\title{
BMJ Open The unmet needs of informal carers of stroke survivors: a protocol for a systematic review of quantitative and qualitative studies
}

\author{
Alexandra M J Denham, ${ }^{1}$ Amanda L Baker, ${ }^{1}$ Neil Spratt, ${ }^{2}$ Ashleigh Guillaumier, ${ }^{1}$ \\ Olivia Wynne, ${ }^{1}$ Alyna Turner, ${ }^{1}$ Parker Magin, ${ }^{1}$ Billie Bonevski ${ }^{1}$
}

To cite: Denham AMJ, Baker AL, Spratt N, et al. The unmet needs of informal carers of stroke survivors: a protocol for a systematic review of quantitative and qualitative studies. BMJ Open 2018;8:e019571. doi:10.1136/ bmjopen-2017-019571

- Prepublication history and additional material for this paper are available online. To view these files, please visit the journal online (http://dx.doi. org/10.1136/bmjopen-2017019571).

Received 14 September 2017 Revised 25 0ctober 2017 Accepted 31 October 2017

Check for updates

${ }^{1}$ School of Medicine and Public Health, Faculty of Health and Medicine, University of Newcastle, Callaghan, New South Wales, Australia ${ }^{2}$ School of Biomedical Sciences and Pharmacy, University of Newcastle, University Drive, Callaghan, New South Wales, Australia

Correspondence to Alexandra M J Denham; alexandra.denham@newcastle. edu.au

\section{ABSTRACT}

Introduction Stroke events deeply affect not only the stroke survivor but also often the quality of life and physical and psychological health of the family and friends who care for them. There is a need for further information about the unmet needs of these informal carers in order to develop support services and interventions. The primary objective of this review is to report and synthesise the research describing the unmet needs of carers of stroke survivors.

Methods and analysis A systematic review of quantitative and qualitative studies that report on the unmet needs of carers will be conducted. The following databases will be searched for relevant articles: MEDLINE, Cumulative Index to Nursing and Allied Health Literature, PsycINFO, EMBASE, Allied and Complementary Medicine Database and Scopus. No publication date constraints will be applied. Studies will be limited to those published in English and conducted among humans. Eligible studies will report on the unmet needs of informal carers of stroke survivors, defined as family members, friends and other unpaid caregivers. Studies which focus on formal, clinical or medical caregivers will be excluded. A narrative synthesis and pooled analysis of the main outcomes will be reported.

Ethics and dissemination This review will be submitted to a peer-reviewed journal. Our findings are expected to provide new insights into the unmet needs of stroke survivors' carers. Knowledge about the unmet needs of carers will inform the development and refinement of interventions and services to address these needs and better support carers of stroke survivors. The findings of this systematic review will be disseminated publicly and in peer-reviewed journals and may be the topic of research presentations.

Trial registration number CRD42017067391.

\section{INTRODUCTION}

\section{Rationale}

As stroke survivors are often cognitively or physically disabled poststroke, many require a carer to support them once discharged from acute care. Approximately $50 \%$ of people who have a stroke will require support from a
Strengths and limitations of this study

- The inclusion of both qualitative and quantitative studies will provide more informative findings on the unmet needs of carers of stroke survivors.

- Our chosen method of analysing and synthesising qualitative and quantitative data has been tested and found effective for systematic reviews.

- The quality of the review will be strengthened by the input and involvement of multiple reviewers at each stage of the review.

- As there are few valid and reliable measures for assessing methodological rigour of qualitative research and mixed-method assessment, there is a risk that flawed studies may bias the results of the systematic review.

- It is also possible that there are relevant non-English studies that will not be included in this review; therefore, these findings may not be generalisable to non-English-speaking populations in which there may be differing cultural unmet needs of carers of stroke survivors.

carer, ${ }^{1}$ and the three most common areas that stroke survivors report needing assistance in are cognitive or emotional tasks, mobility and healthcare. ${ }^{2}$ The Australian Survey of Disability, Ageing and Carers identified that 2.86 million Australians are informal carers, and 26367 of these people have taken on the role of caregiver for someone with stroke as their main condition. ${ }^{3}$ In England and Wales, it is estimated that $10 \%$ of the population are carers, with $60 \%$ of people becoming carers at some point. ${ }^{45}$ In the USA, it is estimated that 4.35 million people are informal carers, ${ }^{6}$ and of those, 2.2 million are people who care for someone who has had a stroke. ${ }^{78}$ These numbers are expected to increase with demographic changes worldwide ${ }^{357}$; therefore, it is becoming increasingly important to recognise the ongoing support needs of carers of stroke survivors. 
There are a number of definitions of 'carers' across stroke studies, ${ }^{9} 10$ generally they are referred to as informal, unpaid or primary carers, or as caregivers. Carers of stroke survivors may be family members, friends or other close individuals who provide physical or emotional support. ${ }^{10}$ Informal carers (defined as people who provide unpaid care, support and/or assistance to someone in need of care) of stroke survivors are predominantly female spouses of the stroke survivor ${ }^{11}$ and often take on a variety of complex tasks and roles, including physical, psychological and daily living support with varying time commitments. The average time spent caring for someone with stroke has been reported to be 41 hours per week. ${ }^{3}$ Furthermore, the replacement financial value of informal care across countries is staggering. If all hours of informal care were replaced with services purchased from formal care providers, the value would be $\$$ A60.3 billion in Australia, ${ }^{3} £ 119$ billion in the UK $^{4}$ and US $\$ 470$ billion in the USA. ${ }^{12}$

Caring for someone who has had a stroke can lead to issues and concerns for the carer, and these issues can also change over time. ${ }^{13}$ For example, when carers must put aside their own needs to care for the stroke survivor, they can experience a sense of loss of autonomy. ${ }^{14}$ Carers often report feeling underprepared to provide practical support following the patient's discharge from hospital and also report feeling excluded from discharge and follow-up plans with health providers. ${ }^{14-16}$ As a result, carers commonly experience a decline in their own physical and mental health and a reduced quality of life. ${ }^{17-19}$ As psychological distress is becoming increasingly common and health outcomes are declining in informal carers ${ }^{20}$ there is a need to gain further understanding of the underlying mechanisms behind the unique unmet needs of informal carers of stroke survivors.

Carer needs often relate to inadequate information and resources, such as receiving information on stroke management and recovery and/or how to contact a healthcare professional who could offer them psychological or emotional support. ${ }^{21}{ }^{22}$ Research suggests that unmet needs result in adverse outcomes, such as increased burden, and depression and anxiety, for carers of those with disabilities and chronic illness. ${ }^{19}{ }^{22-24}$ Greenwood et $a l^{11}$ systematically reviewed qualitative studies investigating the challenges, satisfactions and coping strategies of carers of stroke survivors. The studies in the review described difficulties such as uncertainty, informational/ training needs, and role and relationship changes of carers of stroke survivors. Unmet needs are an extremely important area of research, and it is becoming increasingly important to address these unmet needs to ensure the health and well-being of carers, and the people that they care for.

While there are interventions designed to address the unmet needs of carers of stroke survivors, research suggests that the interventions require further evaluation, development and refinement to target these outcomes. A systematic review conducted by Eldred and Sykes ${ }^{25}$ investigating psychosocial interventions for carers of survivors of stroke found that interventions are failing to address carer depression, loneliness and stress, while there has been some success with interventions that use counselling and education to promote coping and adjustment to the carer role. In these studies, unmet psychological needs (such as depression) were directly reported by carers using validated measures of psychological health outcomes. These findings highlight the need to develop effective psychological interventions targeting the psychosocial functioning of carers of stroke survivors. A systematic review by Aldehaim $e t a l^{26}$ focused on technology-based interventions for carers of stroke survivors. Only one of the five studies eligible for review assessed carer preparedness for the caring role, a known unmet need identified by carers of stroke survivors. ${ }^{27-30}$ That review emphasised the need for interventions to be provided to carers of stroke survivors as soon as possible as a strategy to address unmet needs. The limitations of interventions for carers of stroke survivors include: (1) not being delivered to carers after the stroke survivor is discharged home, (2) not providing appropriate interventions to the changing needs of carers through the stroke survivors' transition from home, to hospital and eventually the community and (3) not targeting appropriate unmet needs outcomes for informal carers, including preparedness and psychosocial functioning, and unique unmet needs for carers of stroke survivors such as support with possible communication and mobility issues.

There are currently no systematic reviews examining the unmet needs of carers of stroke survivors in both qualitative and quantitative studies. Thus, this review aims to:

1. Define and identify the unmet needs of carers of stroke survivors;

2. Define the variables associated with unmet needs of carers of stroke survivors;

3. Report the prevalence of unmet needs identified in quantitative studies;

4. Thematically analyse qualitative data to report meaningful patterns and themes of unmet needs of carers of stroke survivors;

5. Synthesise quantitative and qualitative research on the unmet needs of carers of stroke survivors to guide the development of interventions and services to support carers of stroke survivors.

\section{METHODS AND ANALYSES \\ Study design}

We will conduct a systematic review of studies reporting on unmet needs of informal carers of stroke survivors. These studies will be published in English and have no publication year limit. Qualitative and quantitative studies will be included and synthesised in this review. This systematic review protocol will conform with the Preferred Reporting Items for Systematic Review and Meta-Analysis Protocols (PRISMA-P) ${ }^{31} 32$ and will be reported in accordance with the PRISMA statement. ${ }^{33} 34$ 


\section{Study registration}

Based on the PRISMA-P guidelines, ${ }^{31}$ this systematic review has been registered with the International Prospective Register of Systematic Reviews in July 2017: CRD42017067391.

\section{Eligibility criteria}

\section{Types of studies}

To be eligible for this systematic review, manuscripts must be published in a peer-reviewed journal and report primary data on the unmet needs self-reported by carers of stroke survivors. The needs of carers reported by others (such as the stroke survivors or by health professionals) will be excluded. English language qualitative, quantitative and mixed-methods studies will be included. Intervention studies will be included if they report the baseline estimates of carers' unmet needs.

\section{Participants}

Carers of stroke survivors are defined as the spouse or partner, family members, friends or 'significant others' who provide unpaid physical, practical or emotional support to someone after their stroke event. Stroke survivors will be defined as an individual who has experienced a stroke event. Studies which report on mixed populations, such as reporting on carers' and stroke survivors' unmet needs, will be included in full-text review to investigate if the carers of stroke survivors' data can be extracted separately. Studies will be included if carers provide information related to their unmet needs at any stage of caring for someone following a stroke. Manuscripts that only report on professional carers (doctors, nurses, healthcare providers and others) will be excluded.

\section{Measures}

Quantitative and qualitative studies that report any unmet needs of carers of stroke survivors will be included. Quantitative studies may include self-report surveys. Qualitative studies may include interviews and focus groups. We aim to (1) quantify the proportion of carers of stroke survivors reporting unmet needs in various domains and subcategories, (2) categorise carers of stroke survivors' unmet needs by domain and (3) identify the main variables associated with reporting more unmet needs in carers of stroke survivors.

Unmet needs domains of carers of stroke survivors will be based on Lambert $e t a l^{24}$ which investigated the unmet needs of carers of adult patients with cancer. These include unmet needs relating to changes in role and relationship(s) and informational unmet needs, such as preparedness in caring for someone with a chronic illness and comprehensive knowledge of the condition. As carers of stroke survivors may also have unique needs that differ from being a carer of cancer survivors, other themes that emerge will also be analysed. These needs may include managing the loss of mobility, ${ }^{35}$ language ${ }^{36}$ and $\operatorname{mood} /$ personality changes ${ }^{37}$ as these changes are common following a stroke event.

\section{Search strategy}

'Stroke' terms based on a Cochrane review ${ }^{38}$ have been developed. 'Needs' and 'Partners and Caregivers' terms were be based on a systematic review of unmet needs of partners and caregivers diagnosed with cancer. ${ }^{24}$ In May 2017, a search strategy was developed on the MEDLINE database and then adapted for the other databases. This included medical subject headings and free-text terms using applicable controlled vocabulary. The following electronic databases will be searched: MEDLINE, PsycINFO, EMBASE, Allied and Complementary Medicine Database, Cumulative Index to Nursing and Allied Health Literature, Scopus and Cochrane Database. Reference lists of included studies and relevant systematic reviews will be searched to identify additional studies for potential inclusion in this systematic review. Databases were searched in May 2017, and the search strategy will be conducted weekly across databases to retrieve and screen relevant publications until completion of the systematic review. The search terms for MEDLINE can be found in online supplementary appendix A.

\section{Screening the studies}

Search results will be entered into Endnote folders, and any duplicates will be removed. The online tool Covidence $^{39}$ will be used by reviewers to produce highquality evidence for the systematic review. A flow diagram conforming to PRISMA guidelines ${ }^{33}$ will report the selection process and reasons for exclusion. Two reviewers will independently screen all titles, abstracts, full-text review and data extraction. The two will meet to resolve any issues, and if a decision cannot be made, a third reviewer will be contacted to make the final decision.

\section{Data extraction}

Data will be extracted by one reviewer, tabled and checked for accuracy by another reviewer. The two reviewers will discuss these findings and themes, and if a discrepancy exists and a consensus cannot be reached, a third reviewer will be contacted to make the final decision. Data extracted from the quantitative studies will be extracted and analysed. Data from the qualitative, quantitative and mixed-method studies will be integrated in a systematic way. Common data extracted from the articles will include: study aims, setting, sample characteristics, response rates, study methodology, data analysis, primary outcomes and the unmet needs domains identified. Data extraction tables will be based on Lambert $e t a l$ s research of the unmet needs of carers of adults with cancer. ${ }^{24}$

\section{Quality appraisal}

Qualitative studies

The methodological quality of qualitative studies will be assessed using the Consolidated Criteria for Reporting Qualitative Research (COREQ) ${ }^{40}$ The COREQ was cited by Luker $e t a^{41}{ }^{41}$ in the systematic review of qualitative studies of carers' experiences, needs and preferences during inpatient stroke rehabilitation. This 
quality assessment tool is 32-item tool that promotes comprehensive and rigorous reporting of qualitative studies that use data collection methods of interviews and focus groups. Two reviewers will assess the methodological quality of all studies, and if an agreement cannot be reached, a third reviewer will make the final decision.

\section{Quantitative studies}

The methodological quality of quantitative studies will be assessed based on Greenwood et $a l^{11}$ who performed a systematic review of the quantitative studies focused on factors influencing informal carers of stroke survivors. This quality assessment tool assesses six areas: (1) statement of inclusion or exclusion criteria of carers, (2) clear hypotheses/hypothesis, (3) response rate reported or possible to calculate, (4) multivariate analysis/possible control for confounders, (5) full definition of carer provided (for example, including spouse as the definition); and (6) timing of assessment similar for all participants. Studies are either scored 'Yes' with a value of 1 or 'No' with a value of 0 . Scores are rated on a six-point scale: a score of 6 indicates a maximum score, whereas a score of 0 indicates a minimum score.

\section{Mixed-method studies}

The methodological quality of mixed-method studies will be assessed by using the COREQ ${ }^{40}$ on qualitative components and the methodological assessment developed by Greenwood $e t a l^{11}$ where appropriate regarding quantitative components.

\section{Data analysis}

\section{Qualitative studies}

Qualitative data will be thematically analysed using the qualitative research software NVivo. ${ }^{42}$ Reviewers will develop descriptive themes through interpretation of the meaningful patterns in the qualitative research. Codes for unmet needs domains will be assigned to meaning within the text, and these codes will be compared between studies. Two reviewers will discuss these meanings, and a third reviewer will be consulted if an agreement cannot be reached.

\section{Quantitative studies}

The data analysis of quantitative studies in this review will be based on Lambert et al. ${ }^{24}$ Results across quantitative studies will be pooled, and the prevalence of unmet needs will be compiled. Unmet needs domains will be categorised, and the factors associated within them will be examined. Where reported, the average number of unmet needs will be reported. To facilitate comparison across studies and where appropriate, averages will be standardised from 0 to 100 . For example, where one study reports that the average number of unmet needs is 3 on a maximum of 33 items, a second study might report that it is 7 on a maximum of 44 items. The average will be recalculated on 100, so the average number of unmet needs can be compared across studies. Comparable unmet needs will be combined and clustered into domains. Two reviewers will discuss these outcomes, and a third reviewer will be consulted if a decision cannot be reached.

\section{Mixed-method studies}

The data of mixed-method studies will be analysed appropriately. Qualitative components will be analysed using NVivo $^{42}$ and quantitative components will be based on the data analysis reported by Lambert $e t a .^{24}$

\section{DISCUSSION}

Evidence from this systematic review of unmet needs of carers of stroke survivors will inform the development of interventions and services to address these needs. We will deliver evidence-based recommendations including consideration of the unmet needs of carers of stroke survivors in future research and the refinement and development of interventions and services for this population. These recommendations may improve carer preparedness for their new caregiving role and also assist in supporting carers across all time points of caring for a person who has survived a stroke. These improvements may subsequently improve the quality of life of carers by assisting carers to manage their own needs, mental and physical health, and increase their knowledge in providing physical, emotional and practical care for the stroke survivor. To our knowledge, this will be the first study to systematically synthesise qualitative and quantitative information regarding the unmet needs of carers of stroke survivors.

\section{Strengths and limitations}

The findings of this systematic review are dependent on the quality of original studies that will be reviewed. As there are few valid and reliable measures for assessing methodological rigour of qualitative research $^{43}$ and mixed-method assessment, ${ }^{44}$ there is a risk that flawed studies may bias the results of the systematic review. To minimise this, reviewers will independently review studies and communicate effectively if disagreements occur. It is also possible that there are relevant non-English studies that will not be included in this review. As such, these findings may not be generalisable to non-English-speaking populations, in which there may be differing cultural unmet needs of carers of stroke survivors.

The quality of the review will be strengthened by the input and involvement of multiple reviewers at each stage of the review. Furthermore, the inclusion of both qualitative and quantitative studies will provide more informative findings on the unmet needs of carers of stroke survivors. Our chosen method of analysing and synthesising qualitative and quantitative data has been tested and found effective for systematic reviews. ${ }^{45}$ The findings of this systematic review will be disseminated publicly and in peer-reviewed journals and may be the topic of research presentations. 


\section{Ethics and Dissemination}

Our aim is to publish this systematic review in a peer-reviewed journal. This systematic review will not require ethical approval as data has already published and the analysis is secondary. Our findings are expected to provide new insights into the unmet needs of stroke survivors' carers. Knowledge about the unmet needs of carers will inform the development and refinement of interventions and services to address these needs and better support carers of stroke survivors. Findings will be disseminated widely to clinicians and researchers through journal publication and conference presentations.

Contributors AMJD is the guarantor of the review, performed preliminary data searches and data extraction, conducted quality assessments and drafted the systematic review paper. AMJD, ALB, NS, AG, OW, AT, PM and BB assisted in writing the protocol. All authors contributed to the design and conception of this review, approved the final manuscript and will offer critical revisions for the review manuscript.

Funding This work was supported by a project grant from the National Health and Medical Research Council (NHMRC) of Australia (GNT1045840) and partnership support from the Cancer Council NSW to BB. BB is supported by an Australian NHMRC Career Development Fellowship (GNT1063206) and a Faculty of Health and Medicine, University of Newcastle Gladys M Brawn Career Development Fellowship. AMJD is supported by a Research Training Program (RTP), University of Newcastle PhD Scholarship. ALB is supported by an Australian NHMRC Senior Research Fellowship and a Faculty of Health and Medicine, University of Newcastle Gladys M Brawn Senior Research Fellowship. NS is an Australian NHMRC Career Development/National Heart Foundation (NHF) cofunded by the Career Development/Future Leader Fellow APPS1110629/100827.

Competing interests None declared.

Provenance and peer review Not commissioned; externally peer reviewed.

Open Access This is an Open Access article distributed in accordance with the Creative Commons Attribution Non Commercial (CC BY-NC 4.0) license, which permits others to distribute, remix, adapt, build upon this work non-commercially, and license their derivative works on different terms, provided the original work is properly cited and the use is non-commercial. See: http://creativecommons.org/ licenses/by-nc/4.0/

(c) Article author(s) (or their employer(s) unless otherwise stated in the text of the article) 2018. All rights reserved. No commercial use is permitted unless otherwise expressly granted.

\section{REFERENCES}

1. National Stroke Foundation. National Stroke Audit - rehabilitation services report-2012. Melbourne, Victoria: National Stroke Foundation, 2012.

2. Australian Bureau of Statistics. Disability, ageing and carers, Australia: summary of findings, 2015, 2015.

3. Deloitte Access Economics. 2013. The economic impact of stroke in Australia. Barton: Australian Capital Territory.

4. Carers UK. Valuing carers 2011-calculating the value of carers' London: Support Carers UK, 2011.

5. Carers UK. Facts about caring. Policy briefing 2009; 2009.

6. The National Alliance for Caregiving (NAC), AARP Public Policy Institute. Caregiving in the US, final report. 2015 http:// www.caregiving.org/wp-content/uploads/2015/05/2015_ CaregivingintheUS_Final-Report-June-4_WEB.pdf

7. Stroke Wise. Caregivers for stoke survivors. http://www.strokewise. info/2009/10/caregivers.html

8. American Stroke Association. National family caregivers. http:// www.strokeassociation.org/STROKEORG/LifeAfterStroke/ ForFamilyCaregivers/National-Family-Caregivers-Month_UCM 466774_SubHomePage.jsp.

9. Aziz NA, Pindus DM, Mullis R, et al. Understanding stroke survivors' and informal carers' experiences of and need for primary care and community health services--a systematic review of the qualitative literature: protocol. BMJ Open 2016;6:e009244.

10. Low JT, Payne S, Roderick P. The impact of stroke on informal carers: a literature review. Soc Sci Med 1999;49:711-25.
11. Greenwood N, Mackenzie A, Cloud GC, et al. Informal carers of stroke survivors--factors influencing carers: a systematic review of quantitative studies. Disabil Rehabil 2008;30:1329-49.

12. International Alliance of Carer Organizations. Global carer facts. 2015 http://www.internationalcarers.org/carer-facts/global-carer-stats/\# ednref4.

13. Cameron JI, Naglie G, Silver FL, et al. Stroke family caregivers' support needs change across the care continuum: a qualitative study using the timing it right framework. Disabil Rehabil 2013;35:315-24.

14. Lou S, Carstensen K, Jørgensen CR, et al. Stroke patients and informal carers experiences with life after stroke: an overview of qualitative systematic reviews. Disabil Rehabil 2017;39:301-13.

15. Danzl MM, Harrison A, Hunter EG, et al. 'A lot of things passed me by': rural stroke survivors and caregivers experience of receiving education from health care providers. J Rural Health 2016;32:13-24.

16. Danzl MM, Hunter EG, Campbell S, et al. "Living with a ball and chain": the experience of stroke for individuals and their caregivers in rural Appalachian Kentucky. J Rural Health 2013;29:368-82.

17. Han B, Haley WE. Family caregiving for patients with stroke. Stroke 1999;30:1478-85.

18. Salter K, Zettler L, Foley N, et al. Impact of caring for individuals with stroke on perceived physical health of informal caregivers. Disabil Rehabil 2010;32:273-81.

19. Rigby H, Gubitz G, Phillips S. A systematic review of caregiver burden following stroke. Int J Stroke 2009;4:285-92.

20. Australian Institute of Family Studies. The nature and impact of caring for family members with a disability in Australia; 2008, Research Report No: 16.

21. King RB. The stroke caregiver unmet resource needs scale: development and psychometric testing. J Neurosci Nurs 2015;47.

22. Bakas T, Austin JK, Okonkwo KF, et al. Needs, concerns, strategies, and advice of stroke caregivers the first 6 months after discharge. $J$ Neurosci Nurs 2002;34:242-51.

23. Coombs UE. Spousal caregiving for stroke survivors. J Neurosci Nurs 2007;39:112-9.

24. Lambert SD, Harrison JD, Smith E, et al. The unmet needs of partners and caregivers of adults diagnosed with cancer: a systematic review. BMJ Support Palliat Care 2012;2:224-30.

25. Eldred C, Sykes C. Psychosocial interventions for carers of survivors of stroke: a systematic review of interventions based on psychological principles and theoretical frameworks. $\mathrm{Br} \mathrm{J}$ Health Psychol 2008;13:563-81.

26. Aldehaim AY, Alotaibi FF, Uphold CR, et al. The impact of technologybased interventions on informal caregivers of stroke survivors: a systematic review. Telemed J E Health 2016;22:223-31.

27. Lutz BJ, Young ME, Cox KJ, et al. The crisis of stroke: experiences of patients and their family caregivers. Top Stroke Rehabil 2011;18:786-97.

28. Young ME, Lutz BJ, Creasy KR, et al. A comprehensive assessment of family caregivers of stroke survivors during inpatient rehabilitation. Disabil Rehabil 2014;36:1892-902.

29. Bakas T, Champion V. Development and psychometric testing of the bakas caregiving outcomes scale. Nurs Res 1999;48:250-9.

30. Brereton L. Preparation for family care-giving: stroke as a paradigm case. J Clin Nurs 1997;6:425-34.

31. Moher D, Shamseer L, Clarke M, et al. Preferred reporting items for systematic review and meta-analysis protocols (PRISMA-P) 2015 statement. Syst Rev 2015;4:1

32. Shamseer L, Moher D, Clarke M, et al. Preferred reporting items for systematic review and meta-analysis protocols (PRISMA-P) 2015: elaboration and explanation. BMJ 2015;349:97647.

33. Moher D, Liberati A, Tetzlaff J, et al. Preferred reporting items for systematic reviews and meta-analyses: the PRISMA statement. Ann Intern Med 2009;151:264-9.

34. Liberati A, Altman DG, Tetzlaff J, et al. The PRISMA statement for reporting systematic reviews and meta-analyses of studies that evaluate health care interventions: explanation and elaboration. PLOS Med 2009;6:e1000100.

35. Craig LE, Wu O, Bernhardt J, et al. Predictors of poststroke mobility: systematic review. Int J Stroke 2011;6:321-7.

36. Anderson S, Marlett NJ. The language of recovery: how effective communication of information is crucial to restructuring post-stroke life. Top Stroke Rehabil 2004;11:55-67.

37. Andrewes D, Turner M. Mood and social skills improvement in depressed stroke patients following interpersonal emotional communication training. Brain Impairment 2011;12:67.

38. Lager KE, Mistri AK, Khunti K, et al. Interventions for improving modifiable risk factor control in the secondary prevention of stroke. Cochrane Database Syst Rev 2014:CD009103.

39. Covidence systematic review software. Melbourne, Australia: Veritas health innovation. www.covidence.org. 
40. Tong A, Sainsbury P, Craig J. Consolidated criteria for reporting qualitative research (COREQ): a 32-item checklist for interviews and focus groups. Int J Qual Health Care 2007;19:349-57.

41. Luker J, Murray C, Lynch E, et al. Carers' Experiences, Needs, and Preferences During Inpatient Stroke Rehabilitation: A Systematic Review of Qualitative Studies. Arch Phys Med Rehabil 2017:98:1852-62.

42. QRS International. Helping you discover the rich insights from humanized data. http://www.qsrinternational.com/.
43. Tong A, Flemming K, Mclnnes E, et al. Enhancing transparency in reporting the synthesis of qualitative research: ENTREQ. BMC Med Res Methodol 2012;12:181.

44. O'Cathain A, Murphy E, Nicholl J. The quality of mixed methods studies in health services research. $J$ Health Serv Res Policy 2008;13:92-8.

45. Twyman L, Bonevski B, Paul C, et al. Perceived barriers to smoking cessation in selected vulnerable groups: a systematic review of the qualitative and quantitative literature. BMJ Open 2014;4:e006414. 